\title{
The Behavior of Volatiles on the Lunar Surface ${ }^{1}$
}

\author{
Kenneth Watson, Bruce C. Murray, and Harrison Brown \\ Division of Geological Sciences, California Institute of Technology \\ Pasadena, California
}

\begin{abstract}
Volatiles, and water in particular, have been thought to be unstable on the lunar surface because of the rapid removal of constituents of the lunar atmosphere by solar radiation, solar wind, and gravitational escape. The limiting factor in removal of a volatile from the moon, however, is actually the evaporation rate of the solid phase, which will be collected at the coldest points on the lunar surface. We present a detailed theory of the behavior of volatiles on the lunar surface based on solid-vapor kinetic relationships, and show that water is far more stable there than the noble gases or other possible constituents of the lunar atmosphere. Numerical calculations indicate the amount of water lost from the moon since the present surface conditions were initiated is only a few grams per square centimeter of the lunar surface. The amount of ice eventually detected in lunar 'cold traps' thus will provide a sensitive indication of the degree of chemical differentiation of the moon.
\end{abstract}

\section{INTRODUCTION}

Previous analyses of the behavior of volatiles on the lunar surface [Spitzer, 1952; Kuiper, 1952; Urey, 1952; Öpik and Singer, 1960; Vestine, 1958] have all indicated that volatiles could not survive there for extended periods of time, and that water is particularly unstable because of its low molecular weight and ease of ionization. These investigations did not, however, recognize that the amount of any volatile in the vapor phase on the moon-and hence its mass removal rate from the moon-is determined by the temperature of the solid phase at the coldest place on the lunar surface. This was recently pointed out by the authors [Watson, Murray, and Brown, 1961]. We have now developed a detailed theory of the behavior of volatiles on the lunar surface which takes vapor pressure equilibrium into account, and from it we show that water is actually by far the most stable of the naturally occurring volatile substances that might conceivably have been released at some time on the lunar surface. We show further that the total amount of water that could have been removed since the time the moon's surface conditions first evolved to essentially their present characteristics is quite small. Thus the present amount of lunar ice must bear a close relationship to the total amount of water ever present

1 Contribution 1048, Division of Geological Sciences, California Institute of Technology. on the lunar surface over the above time period. Conversely, the absence of lunar ice would indicate an extremely small amount of chemical differentiation of the lunar mass.

We will first develop the theory of the behavior of volatiles on the lunar surface, and then investigate the best numerical values of the parameters of our model to use in numerical calculations.

\section{Theory of Migration and Trapping of Volatiles and Their Escape from the Lunar Surface}

In this section we shall develop a theory of the behavior of volatile substances on the lunar surface which is based on two premises: (1) that the lunar atmosphere is so rarefied that molecular transport in the vapor phase can be described purely in terms of dynamical trajectories; and (2) that there are permanently shaded areas (cold traps) with temperatures at least as low as $120^{\circ} \mathrm{K}$. It will be convenient to discuss first the steady loss from the cold traps, and, second, losses of any newly liberated volatiles during migration to the cold traps. By combining these results we will derive the equation governing the total mass removal rates of volatiles from the lunar surface.

2.1. Steady-state loss of volatiles from the cold trap. Once any substance condenses in a permanently shaded area, it will be subject to a continual evaporation loss, which may be 


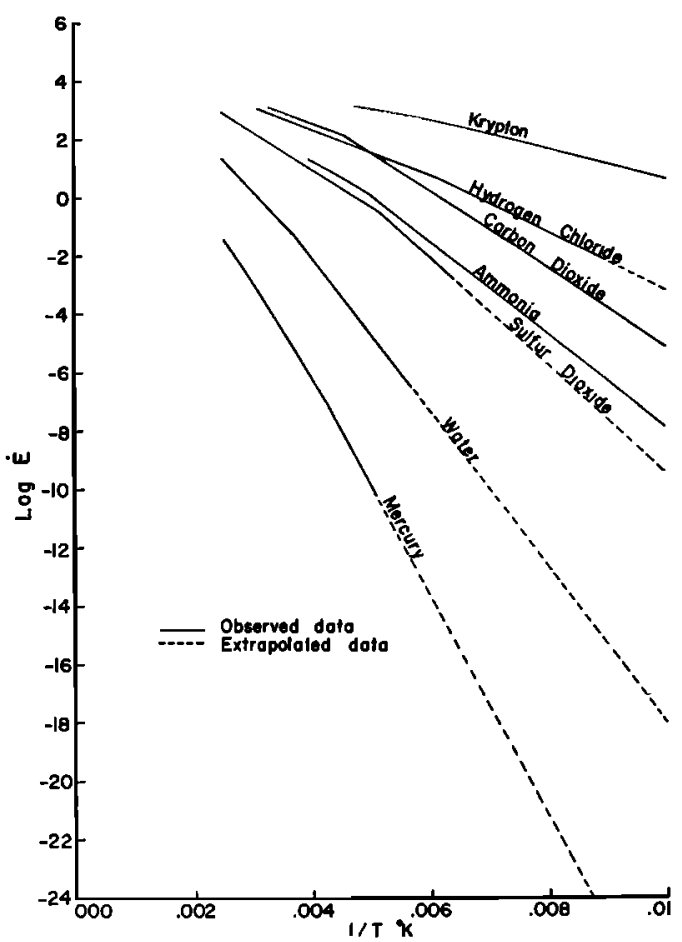

Fig. 1. Evaporation rates.

balanced in part by condensation back into the cold trap from the atmosphere. The mass loss rate from the cold traps, $\dot{m}_{s}$, is simply the difference between evaporation and condensation rates:

$$
\dot{m}_{a}=K A(\dot{E}-\dot{C})
$$

where

$K=$ fraction of lunar surface that is permanently shaded.

$A=$ total lunar surface area in square centimeters.

$\dot{E}=$ evaporation rate of the substance at the surface temperature of the cold trap, taken to be $120^{\circ} \mathrm{K}$ as an upper limit for the moon in grams per square centimeter per second.

$\dot{C}=$ condensation rate of the substance in question back into cold trap areas in grams per square centimeter per second.

The fraction of the lunar surface in permanent shadow, $K$, will be estimated later in this paper to be about $5 \times 10^{-8}$. The evaporation rate is a characteristic physical property of any solid phase that depends only on the surface temperature of that solid. The evaporation rate could be observed directly if the vapor were removed as fast as it formed, i.e., by eliminating condensation. On the other hand, evaporation and condensation rates are equal when the vapor is saturated, if the accommodation coefficient is unity. Accordingly, the equilibrium vapor pressure present over a solid surface can be used to estimate the characteristic evaporation rate at that temperature. The maximum evaporation rate can therefore be expressed as follows [Estermann, 1955].

$\dot{E}=p \sqrt{\frac{\mu}{2 \pi R T}}=4.374 \times 10^{-5} \times p \sqrt{\frac{\mu}{T}}$

where

$\dot{E}=$ mass loss in grams per square centimeter per second.

$p=$ vapor pressure in dynes per square centimeter.

$\mu=$ molecular weight.

$T=$ absolute temperature.

$R=$ gas constant.

In order to obtain values of $\dot{E}$ in the vicinity of $120^{\circ} \mathrm{K}$ for a number of naturally occurring volatile substances, the appropriate equilibrium vapor pressures have been extrapolated to low temperatures for the present analysis [International Critical Tables, 1928]. The experimental data are of the form $\log p=-A / T+B(A$ and $B$ are constants characteristic of the substance), and linear extrapolation is reasonable for our purposes, assuming that no solid phase changes occur at these pressures. For ice, this assumption is experimentally validated by the work of Bridgman [1957].

The resulting maximum evaporation rates for various volatiles as a function of temperature are plotted in Figure 1.

The condensation rate $\dot{C}$, unlike $K, A$, and $\dot{E}$, is not a simple characteristic property of either the moon or of a particular substance because it also depends on the escape rate from the lunar atmosphere and the accommodation coefficient in the cold traps. However, it is possible to treat the migration of molecules on the lunar surface in terms of a simple probability model, provided that the average trajectory jump length of the molecules is comparable to or larger than the average size and separation of the cold traps. 
The derivation of $\dot{C}$ in this way is presented now. Later we will demonstrate that the moon does indeed satisfy the restriction regarding average jump length vs. size and separation of cold traps.

The following parameters will be used:

$t=$ jump time, which is a function of the velocity distribution at the surface as well as of the molecular weight and the lunar surface gravity. $Z$ and $\bar{D}$ are mean jump time and mean jump length, respectively.

$\alpha=$ probability for a molecule to escape from the lunar atmosphere on a single jump.

$K=$ fraction of the lunar surface occupied by permanently shaded areas.

$a=$ accommodation coefficient, the probability that an incident molecule will stick to the surface.

The processes by which the molecules transport themselves through the rarefied atmosphere and either escape or become trapped in the permanently shaded areas are complicated in detail. For our purposes it is sufficient to realize that the fraction of the molecules that condense on a surface, as opposed to the fraction that are reflected, is controlled by the microscopic roughness and chemical nature of the surface, by the surface temperature, and by the molecular weight of the impacting molecules. A discussion of this can be found in Loeb [1927], Langmuir [1917], and Knudsen [1910]. We shall assume that the temperature and microscopic roughness of the cold-trap surfaces are sufficient to ensure that the accommodation coefficient is close to unity. We will, however, consider the effect of varying $a$ when numerical calculations for the trapped fraction are performed. It is also reasonable from the above to assume that molecules rebounding from the warm surfaces will approach the surface temperature after a few jumps, regardless of the velocity distribution at the source.

It is apparent that those molecules captured in temporarily shaded areas, such as the dark side, cannot escape until the host area heats up. Hence, we can neglect the effect of these temporarily shaded areas in the solution of the problem, and consider only those molecules actually moving at any instant of time.

We shall further assume, to avoid unnecessary complexity, that the reflection and emission of molecules from the lunar surface can be adequately described by a cosine distribution. The average jump time $Z$, and the average jump distance $\bar{D}$, are, therefore:

$$
\begin{gathered}
Z=2 \times \frac{.866 \times V}{g} \\
\bar{D}=\frac{1}{2} V t
\end{gathered}
$$

where

$$
\begin{aligned}
& V=\text { rms velocity }=\sqrt{3 R T / \mu} . \\
& g=\text { surface gravitational acceleration } \\
& R=\text { gas constant. } \\
& T=\text { effective surface temperature }\left({ }^{\circ} \mathrm{K}\right) \\
& \mu=\text { molecular weight of volatile. }
\end{aligned}
$$

We now examine the implications of our assumptions. If $\bar{D}$ is at least comparable to the size and separation of the permanently shaded areas, we can presume that for any individual jump the probability of landing on a permanently shaded area is proportional only to the total fractional area of permanent shade $K$, and does not depend on the location of the beginning point of the jump. Since $\alpha$ represents the escape probability during an individual jump, the probability of a particle landing on a coldtrap surface and not escaping is $K(1-\alpha)$. Finally, from our discussion of the coefficient of accommodation, it is clear that of those particles landing on a cold trap a fraction $a$ must remain trapped. Hence, on any individual jump: probability of a particle being trapped $=K$ $(1-\alpha) a$

where

$$
\begin{aligned}
& \alpha=\text { probability of a particle escaping on a } \\
& \text { single jump. }
\end{aligned}
$$

Now since we assume that the jumps are uncorrelated, the above discussion must apply for all particles during a single jump. Hence $\alpha=1-\exp (-Z / \tau)$ where $\tau$ is a characteristic decay time for many-particle jumps. Therefore, the fraction, $\gamma$, of molecules that become permanently trapped on any jump compared to the total that either escape or are trapped on the jump is

$$
\gamma=\frac{K a(1-\alpha)}{K a(1-\alpha)+\alpha}
$$

However, the net number of particles in the 
lunar atmosphere must remain constant for a constant escape probability $\alpha$, and certainly does not change significantly during the time of a single jump. Hence, the evaporation rate must be exactly equal to the condensation rate plus the escape rate. Therefore,

or

$$
\dot{E}=\dot{C}+(1-\gamma) \dot{E}
$$

$$
\dot{C}=\gamma \dot{E}
$$

and, from equations 1 and 6

$\dot{m}_{s}=K A \dot{E}(1-\gamma)=(1-\gamma) \dot{m}_{s(\max )}$

and from equation 5 :

$$
\dot{m}_{s}=K A \dot{E}\left(\frac{\alpha}{K a(1-\alpha)+\alpha}\right)
$$

This relationship clearly demonstrates important physical restrictions on $\dot{m}_{s}$, the mass loss rate. For very large values of the decay time $\tau$, the probability of escape $\alpha$, and $\dot{m}_{s}$, are essentially zero. Thus, from equation $1, \dot{E}$ is equal to $\dot{C}$, and the partial pressure of a particular constituent in the lunar atmosphere is just the equilibrium vapor pressure corresponding to the temperature of the cold trap. For very small values of $\tau$ (rapid escape), $\alpha$ approaches 1 , $\dot{m}$, becomes $K A \dot{E}=\dot{m}_{\text {s (max) }}$, and the partial pressure drops to zero. It is particularly important to note that the value $K A \dot{E}=\dot{m}_{a}$ (max) is an absolute upper limit for the rate of removal of a condensed volatile from all the cold traps on the moon. As Figure 2 illustrates, $\dot{m}_{s}$ is limited by $K a$ and by the evaporation rate of a particular volatile at $120^{\circ} \mathrm{K}$, for example, rather than by the escape probability. For this reason, the evaporation rate data of Figure 1 are a much more meaningful guide to stability of volatiles on the lunar surface than are properties such as molecular weight, ionizability, etc., that enter into escape computations.

2.2. Transient loss of newly liberated volatiles during migration to cold traps. We must also consider the behavior of any new volatile material that might be liberated at an arbitrary point on the lunar surface either sporadically or steadily. Let us define

$\dot{m}_{f}=$ mass influx of newly liberated volatiles for the entire lunar surface, in grams per second. This can be considered to be either a smooth function or a series of random impulses of arbitrary amounts.

$\dot{m}_{f l}=$ mass loss rate by escape during random migration of the molecules from the source point to a cold trapping surface, in grams per second.

If we assume again that the end points of molecular jumps are uncorrelated with beginning points, then the previous derivation for the condensation rate applies to the present problem as well. In particular, the fraction of newly liberated molecules that get trapped on their first jump, or on any subsequent jump, is simply $\gamma=[K a(1-\alpha)] /[K a(1-\alpha)+\alpha]$. Hence

$$
\dot{m}_{f l}=(1-\gamma) \dot{m}_{f}
$$

2.3. Basic equations describing mass losses. Since the mass rate of removal by evaporation from the cold traps is independent of the rate of liberation of any new volatile materials from the lunar surface, the two loss rates $\dot{m}_{\mathrm{s}}$ and $\dot{m}_{f l}$ are independent. Therefore, from equations 7 and 9:

$$
\begin{aligned}
\dot{m}=\dot{m}_{s} & +\dot{m}_{f l} \\
& =(1-\gamma)\left(\dot{m}_{s(\max )}+\dot{m}_{f}\right)
\end{aligned}
$$

when $\dot{m}$ is the total mass loss rate from the lunar surface in grams per second and the accumulated loss over an interval of time $T_{2}-T_{1}=\Delta T$ is simply

$$
\begin{aligned}
& \Delta M=\int_{T_{1}}^{T_{2}} \int \dot{m} d t=(1-\gamma) \\
& \cdot\left(\dot{m}_{s(\max )}+\dot{m}_{f}\right) \Delta T
\end{aligned}
$$

The mass loss from cold traps only can be expressed as

$$
\begin{gathered}
\dot{m}_{c}=\gamma \dot{m}_{f}-\dot{m}_{s} \\
=\gamma \dot{m}_{f}-(1-\gamma) \dot{m}_{s(\max )} \\
m_{c}(t)=m_{c}(0)+\gamma t \dot{m}_{f} \\
\quad-(1-\gamma) t \dot{m}_{s(\max )}
\end{gathered}
$$

In the next section we will consider the actual value of the parameters of the equation, and compute $m_{c}(t)$ and $t$ comparable to the lifetime of the moon.

\section{Applications to the Lunar Surface}

3.1. Introduction. It is first necessary to show that the environment of the lunar surface 


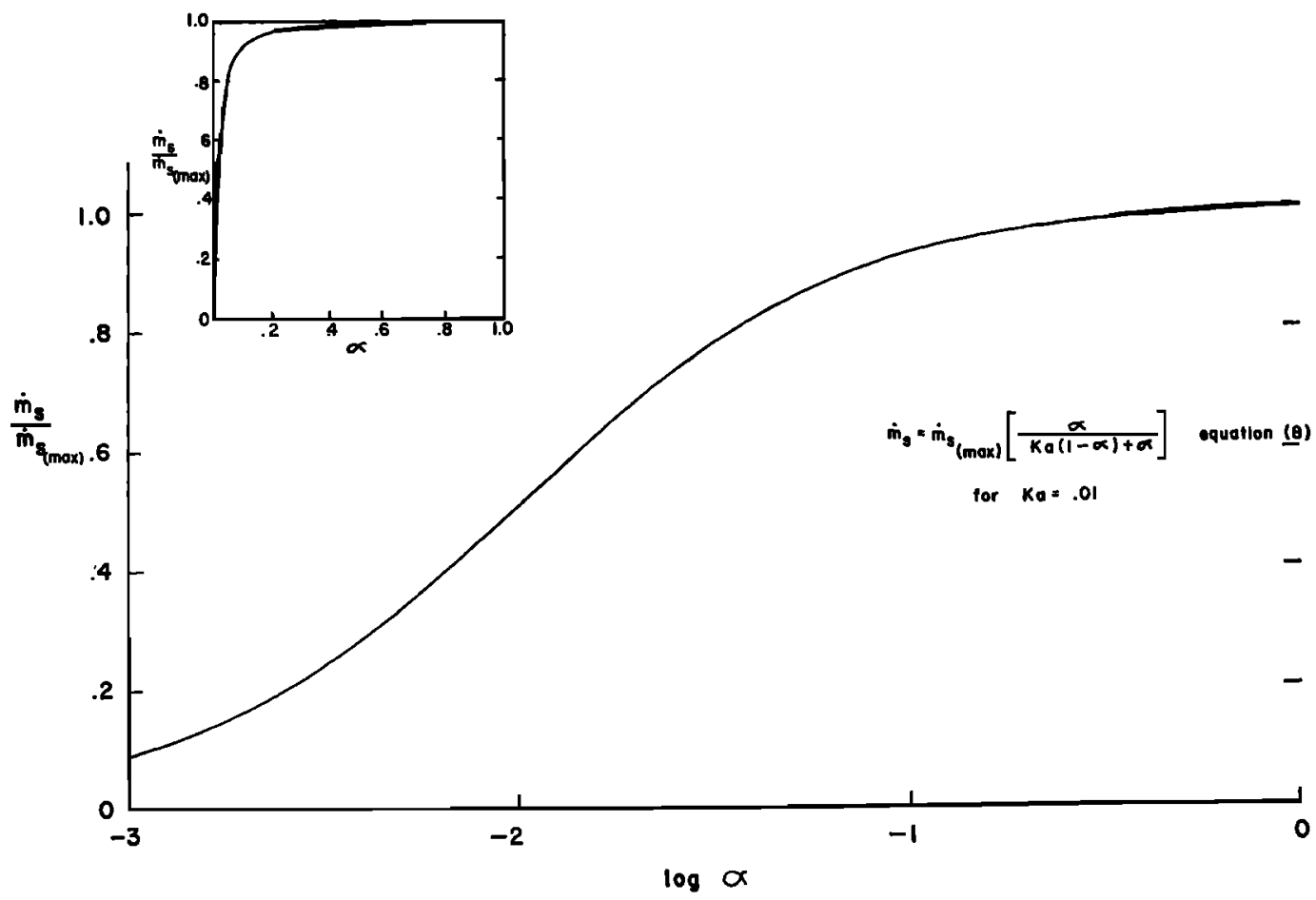

Fig. 2. Mass loss rate vs. escape probability.

satisfies the two basic premises of our model. The existence of a rarefied atmosphere has been proven observationally by Dollfus [1952], Elsmore and Whitfeld [1955], and Opik [1955].

The most recent studies by Dollfus [1956] of polarization and by Elsmore [1957] of radio occultations have verified the previous results and indicate that the lunar atmosphere is extremely rarefied. The maximum density consistent with the measurements of Elsmore yields a mean free path of the order of $10^{4} \mathrm{~km}$, which is sufficient to justify the use of ballistic trajectories for the mechanics of molecular transport in the lunar atmosphere, since the average jump length for molecules in equilibrium with the lunar surface is of the order of $10^{2} \mathrm{~km}$.

Infrared studies of the lunar surface by Menzel [1923], Pettit and Nicholson [1930], and Sinton [1955] indicate that the shaded areas of the lunar surface have maximum temperatures of the order of $120^{\circ} \mathrm{K}$. Thus $120^{\circ} \mathrm{K}$ represents an upper limit for permanently shaded areas; much lower temperatures would be expected if the surface temperature resulted principally from the balance on that surface between an interior heat flow and radiative cooling. Simple computations indicate that the low conductivity of the dust layer as proposed by Jaeger [1953] and Wesselink [1948] will prevent even small areas of permanent shade from being heated up during the lunar day by horizontal conduction from adjacent illuminated areas.

The next two sections are devoted to a discussion of reasonable and limiting numerical values for $K$, the fraction of permanent shade, and $\alpha$, the probability of escape. Using these values, the mass removal rates for common volatile substances will be compared, and we will conclude that water, in the form of ice, is the only common volatile that could be stable for a period of time comparable to age of the moon. We then discuss possible sources for the liberation of water from the lunar surface. Finally, a discussion is presented of the validity of the uncorrelated model as a description of migration on the actual lunar surface.

3.2. The amount and distribution of permanently shaded areas on the lunar surface. The obliquity of the moon's axis of rotation with respect to the ecliptic is only $1^{\circ} 32^{\prime}$ [Allen, 1955]; there is 


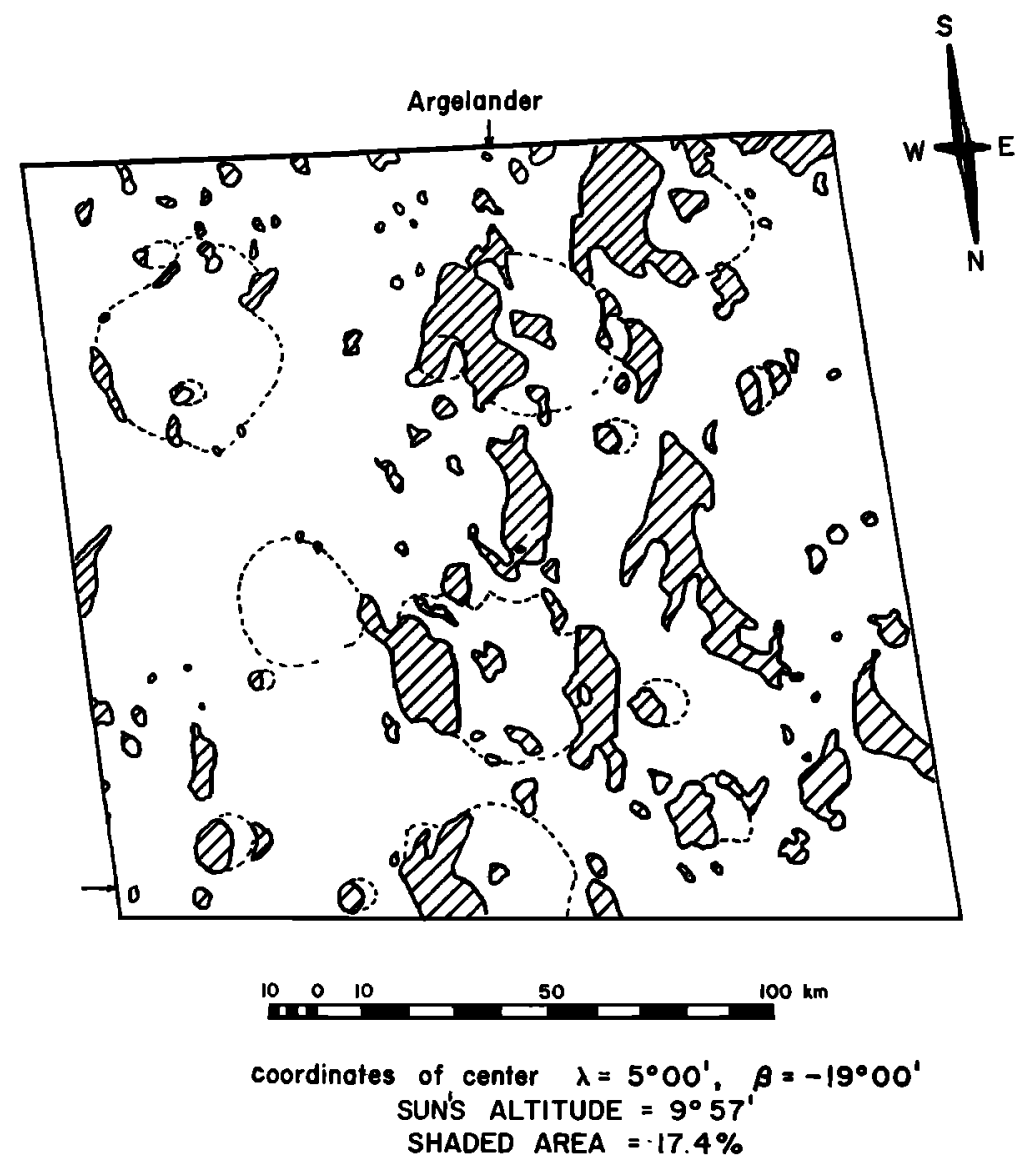

Fig. 3. Tracing of the shaded areas near the crater Argelander for the sun's altitude of $9^{\circ} 57^{\prime}$.

virtually no seasonal variation on the moon. Accordingly, many steep-walled craters at high lunar latitudes will be permanently shaded on the part of the wall and bottom of the crater nearest the equator. However, any terrestrial observation of the moon is necessarily made from a selenodetic direction that is also occupied by the sun at one time or another; hence, it is impossible to study the permanently shaded areas directly from the earth. One may attack the problem, however, in at least two indirect ways: (1) define some necessarily crude analytical model of a crater, compute its permanently shaded area as a function of latitude, and use some distribution function for lunar craters to arrive at an estimate of $K$, the fraction of permanently shaded craters for the whole lunar surface, or (2) use photographs of upland areas near the equator to obtain estimates of the actual roughness in the polar areas, and, then, by measuring the variation in per cent shaded area as a function of the sun's elevation, estimate $K$. The first technique was applied by Watson, Murray, and Brown [1961] in order to get an absolute lower limit of $K=10^{-4}$. The second approach, which is discussed briefly in the present paper and will be published elsewhere, yields an actual estimate of $K=5 \times 10^{-3}$ (rather than just a lower limit).

Figure 3 is a tracing of the shaded areas in a small part of the lunar surface sufficiently near $\lambda=0, \beta=0$, so that any bias in projection is small. The character of the topography in the test area is quite similar to that in the polar areas: both display a dense array of craters of random-size distribution. Mountains, valleys, and other linear topographic features are almost entirely restricted to the borders of the marias 
TABLE 1. Estimation of Permanently Shaded Area on Lunar Surface

\begin{tabular}{|c|c|c|c|c|c|}
\hline $\begin{array}{l}\text { Latitude } \\
\text { Zone, } \\
\text { deg }\end{array}$ & $\begin{array}{c}\text { Total Lunar } \\
\text { Surface, } \\
\%\end{array}$ & $\begin{array}{c}\text { Upland in } \\
\text { Latitude Zone, } \\
\%\end{array}$ & $\begin{array}{c}\text { Total } \\
\text { Upland, } \\
\%\end{array}$ & $\begin{array}{c}\text { Shaded Area } \\
\text { at Noon, } \\
\%\end{array}$ & $\begin{array}{l}\text { Pernianently } \\
\text { Shaded Area, \% } \\
\text { (for reduction } \\
\text { factor } 0.5 \text { ) }\end{array}$ \\
\hline $90-80$ & 1.53 & 100 & 1.53 & 27.5 & 0.21 \\
\hline $80-70$ & 4.54 & 100 & 4.54 & 85 & 0.19 \\
\hline $70-60$ & 7.41 & 94.5 & 7.00 & 2.2 & 0.08 \\
\hline $60-50$ & 10.04 & 78.5 & 7.88 & 10 & 003 \\
\hline $50-40$ & 12.36 & 77.5 & 9.57 & & \\
\hline $40-30$ & 14.28 & 67.5 & 9.63 & & \\
\hline $30-20$ & 15.76 & 57.0 & 8.99 & & \\
\hline $20-10$ & 16.78 & 48.0 & 8.05 & , & \\
\hline \multirow[t]{2}{*}{$10-0$} & 17.30 & 47.0 & 8.13 & & \\
\hline & & Total & 65.3 & \multicolumn{2}{|c|}{$\begin{array}{l}\text { Total } \\
K=5 \times 10^{-3}\end{array}$} \\
\hline
\end{tabular}

and, consequently, can be ignored when considering high latitudes where the permanently shaded areas are concentrated.

The relative proportion of upland as a function of latitude for the visible face of the moon is shown in column four of Table 1 . The percentage of the test area shaded at different elevation angles of the sun has been measured from other photographs of the same area at different lunar phases. Since the per cent shaded area of any crater on the equator at a sun's elevation of, for instance, $20^{\circ}$, is about equal to the shaded area that same crater would exhibit at local noon time if it were at a latitude of $90^{\circ}-20^{\circ}=70^{\circ}$, it is possible to use the measurement of per cent shading vs. sun's elevation for an equatorial test area as a good estimate of the per cent shaded area at noon time of similar topography at higher latitudes. The data from the photographs are plotted in the fifth column of Table 1 in this manner, except that the $1^{\circ} 32^{\prime}$ annual variation of the sun's elevation has been taken into account. The annual variation, of course, is equivalent to increasing the sun's elevation at all latitudes by that amount. For any crater the minimum shaded area occurs at noon time, but this shadow then is still larger than the permanently shaded area. This is because the sun illuminates a part of the border of the area covered by the noon-time shadow at other times during the lunar day, even though the elevation angle is less than at noon time. We take a value of 0.5 as an average reduction factor for lunar craters. The estimated permanently shaded area vs. latitude as a percentage of the total lunar surface area is then merely the product of columns 4 and 5 , reduced by the $1 / 2$ factor.

The main source of error leading to an overestimate of $K$ would arise from a poor choice of the reduction factor; $K=10^{-s}$ would seem to be an absolute lower limit. Similarly, the facts that (1) there are less maria on the reverse side of the moon than on the visible one Barabashov, Michailov, and Lipski [1961], and (2) that craters less than $1 \mathrm{~km}$ in diameter have effectively been ignored owing to limited resolution, might suggest that the true value of $K$ is higher than $5 \times 10^{-3}$. An upper value of $K=10^{-2}$ seems appropriate. Accordingly, we conclude that $K$ lies between 0.1 and 1 per cent, most likely about 0.5 per cent.

3.3. Escape mechanisms. The most important escape mechanisms proposed so far are ionization, solar wind collision, and gravitation.

Opik and Singer [1960] have calculated the half-life of krypton in an exosphere in contact with the lunar surface under solar ultraviolet radiation. They show that incident radiation will ionize the atmosphere and also raise the electrostatic potential on the lunar surface by photoionization of silicates. By calculating the screening length associated with this potential and comparing it with the characteristic 'scale height' of the atmosphere they calculated a half-life of $4.3 \times 10^{10}$ secs ( $\tau=6 \times 10^{10}$ seconds). Opik and Singer (private communication) have also estimated the decay time for photodissociation of water vapor in the lunar atmosphere by 
TABLE 2. Escape Parameters

\begin{tabular}{|c|c|c|c|c|c|c|}
\hline \multirow[b]{2}{*}{ Volatile } & \multirow[b]{2}{*}{$\mu$} & \multirow[b]{2}{*}{$\begin{array}{c}\bar{D} \\
\mathrm{~km}\end{array}$} & \multirow[b]{2}{*}{$\begin{array}{c}\bar{t} \\
\sec \end{array}$} & \multicolumn{3}{|c|}{ Escape Probability, $\alpha$} \\
\hline & & & & $\begin{array}{c}\text { Solar } \\
\text { Radiation }\end{array}$ & $\begin{array}{l}\text { Solar } \\
\text { Wind }\end{array}$ & Gravitation \\
\hline Mercury & 200 & 26 & 133 & & $1 \times 10^{-3}$ & $5 \times 10^{-74}$ \\
\hline Krypton & 83 & 62 & 206 & $2 \times 10^{-9}$ & $2 \times 10^{-3}$ & $2 \times 10^{-30}$ \\
\hline Sulfur dioxide & 64 & 61 & 236 & & $2 \times 10^{-3}$ & $3 \times 10^{-23}$ \\
\hline Carbon dioxide & 44 & 118 & 285 & & $3 \times 10^{-3}$ & $7 \times 10^{-16}$ \\
\hline Hydrogen chloride & 36 & 142 & 315 & & $3 \times 10^{-3}$ & $4 \times 10^{-13}$ \\
\hline Water & 18 & 287 & 446 & $4 \times 10^{-3}$ & $4 \times 10^{-3}$ & $2 \times 10^{-6}$ \\
\hline Ammonia & 17 & 304 & 458 & & $5 \times 10^{-3}$ & $4 \times 10^{-6}$ \\
\hline
\end{tabular}

solar radiation to be of the order of $10^{5}$ seconds or less, but the relevant calculations have not yet been published.

Herring and Licht [1959] have estimated the reduction in a lunar atmosphere due to the influx of the high-energy protons in the solar wind. Since the protons are traveling with an assumed velocity of $10^{8} \mathrm{~cm} / \mathrm{sec}$ they have an incident energy of about $5 \mathrm{kev}$. Inasmuch as the energy necessary for escape of even the heavy volatiles from the lunar atmosphere is less than $5 \mathrm{ev}$ it is apparent that the energy transferred by proton-molecule impact is more than sufficient to cause escape. The molecular diameter of the volatiles is $3 \times 10^{-8} \mathrm{~cm}$, hence the cross section for impact is about $10^{-16} \mathrm{~cm}^{2}$. For a solar wind density of $10^{2}$ particles $/ \mathrm{cm}^{3}$, the decay time is $\left(10^{-15} \times 10^{2} \times 10^{8}\right)^{-1}=10^{5}$ seconds.

The least rapid rate of removal is by direct escape of that fraction attaining thermal velocities greater than the escape velocity. Spitzer [1952] has derived the equations for the escape time. We shall assume that the temperature at the base of the exosphere is the lunar surface temperature of $400^{\circ} \mathrm{K}$. Then

$$
\tau=\frac{\sqrt{6 \pi}}{3 g} \cdot V \frac{e^{Y}}{Y}
$$

where

$$
Y=\frac{3 V_{\infty}^{2}}{2 V^{2}}
$$

and

$V_{\infty}=$ escape velocity.

$V=$ rms velocity associated with $400^{\circ} \mathrm{K}$.

For water, $\tau=2.5 \times 10^{8}$ seconds.

Table 2 gives $\alpha$, the escape probability, for the mechanisms we have discussed, as well as the values for $\bar{t}$, the average jump time, and $\bar{D}$, the average jump length for selected volatiles.

3.4. Mass loss rates of water and other volatiles. The mass loss rate per unit lunar surface area can be computed from equation 8 . We shall use the value for $K$ of $5 \times 10^{-3}$, and, assuming $a=1$, we use Table 2 and the evaporation rates (Fig. 1) to compute $\dot{m}_{s}$. Our assumption that $a$ is unity for ice is justified by the work of Tschudin [1946] who measured evaporation rates of ice in the range $-60^{\circ} \mathrm{C}$ to $-85^{\circ} \mathrm{C}$ and determined a value of .94 for $a$. The results are presented in Table 3 and illustrated graphically in Figure 4.

It is apparent that mechanical escape is far too inefficient to be considered a significant loss mechanism. A comparison of the other loss rates leads us to the conclusion that only water and mercury could remain trapped for an appreciable fraction of lunar history. Because of the extremely low abundance of free mercury on the earth's surface, it is reasonable to neglect it from further lunar consideration. Accordingly, water is the only volatile possibly retained in significant quantities on the lunar surface. We will use the mass loss rate associated with photodissociation as a reasonable estimate for the escape of water.

The total mass loss rates of water are computed for two values of $\alpha$ : a 'reasonable' one corresponding to $\tau=10^{5}$ seconds, and a maximum one of $\tau=0$ seconds (i.e., zero condensation), where values for the latter will be given in brackets following the values associated with the reasonable estimate.

In one billion years the mass loss rate from the cold traps is $4 \mathrm{~g} / \mathrm{cm}^{2}{ }_{m}\left(9 \mathrm{~g} / \mathrm{cm}^{2}\right)\left(\mathrm{cm}_{m}^{2}\right.$ refers to the whole lunar surface). This is equivalent to 
TABLE 3. Mass Loss Rate Per Unit Area of the Lunar Surface $\mathrm{m}_{x} / \mathrm{A}\left(\mathrm{g} / \mathrm{cm}^{2} / \mathrm{sec}\right)$

\begin{tabular}{lllll}
\hline \multicolumn{1}{c}{ Volatile } & $\begin{array}{c}\text { Solar } \\
\text { Radiation }\end{array}$ & $\begin{array}{c}\text { Solar } \\
\text { Wind }\end{array}$ & Mechanical & $\begin{array}{c}\text { Maximum } \\
\text { limited by } \\
\text { evaporation }\end{array}$ \\
\hline Mercury & & $6 \times 10^{-26}$ & $2 \times 10^{-96}$ & $3 \times 10^{-25}$ \\
Krypton & $1 \times 10^{-7}$ & $9 \times 10^{-2}$ & $1 \times 10^{-28}$ & $3 \times 10^{-1}$ \\
Sulfur dioxide & & $1 \times 10^{-9}$ & $2 \times 10^{-29}$ & $4 \times 10^{-9}$ \\
Carbon dioxide & & $1 \times 10^{-6}$ & $1 \times 10^{-18}$ & $1 \times 10^{-5}$ \\
Hydrogen chloride & $1 \times 10^{-16}$ & $1 \times 10^{-4}$ & $2 \times 10^{-14}$ & $3 \times 10^{-4}$ \\
Water & $1 \times 10^{-16}$ & $1 \times 10^{-19}$ & $3 \times 10^{-16}$ \\
Ammonia & & $3 \times 10^{-8}$ & $5 \times 10^{-11}$ & $6 \times 10^{-8}$ \\
\hline
\end{tabular}

a removal of a total of 8 meters (18 meters) of ice from the cold traps. We now calculate the necessary amount of new water that would have been liberated from the whole lunar surface to replenish the loss from the cold traps. From equation 5 the mass addition rate to the cold traps owing to liberation of new volatiles is $\gamma \dot{m}_{f}$. Hence, for no net mass loss from the cold traps,

$$
\gamma \dot{m}_{f}=\dot{m}_{s}
$$

and, from equations 7 and 15

$$
\dot{m}_{f}=\frac{1-\gamma}{\gamma} \dot{m}_{s(\max )}
$$

Using our 'reasonable' estimate for $\dot{m}_{s}$, the required liberation rate is $7 \mathrm{~g} / \mathrm{cm}^{2}$ / billion years.

3.5. Production of water on the lunar surface. Virtually all known silicate materials that fall upon the earth from space appear to contain water in varying amounts, the greater part of which is probably chemically bound. The hypersthene, bronzite, and enstatite chondrites contain very low concentrations of water, starting at about 0.02 per cent, averaging about 0.25 per cent, and seldom exceeding 0.5 per cent. There is always the uncertainty that some of the observed water might be of terrestrial origin, but water does appear to be present in fresh falls which have been analyzed shortly after recovery. Concentrations range upward to as high as 20 per cent in some carbonaceous chondrites. In the latter cases there is little question that the water is extraterrestrial. These meteorites represent some 3 per cent of all observed falls.

The visible water now on the surface of the earth, which appears to have been present originally in chemically bound form, amounts to somewhat over $280 \mathrm{~kg} / \mathrm{cm}^{2}$ of the earth [Rankama and Sahama, 1950]. Approximately $6 \mathrm{~kg}$ of water per square centimeter of the earth appears to be bound in the sediments and, judging from the observed water contents of basaltic materials, an additional $50 \mathrm{~kg} / \mathrm{cm}^{2}$ may still reside in the crust above the Mohorovicic discontinuity. Some $30 \mathrm{~kg}$ of water per square centimeter has perhaps been lost over geologic time as the result of dissociation in the atmosphere followed by the escape of hydrogen. Thus, if the earth is welldegassed below the Mohorovicic discontinuity, the primitive earth would have contained some $370 \mathrm{~kg}$ of water per square centimeter. On this basis the original earth material would have contained about 0.03 per cent water, an amount comparable to the apparent abundance of bound water in many chondrites of the noncarbonaceous variety.

Had primitive lunar material contained an equivalent concentration of water, the moon would have contained originally an amount of water equal to about $60 \mathrm{~kg}$ of water per square centimeter of the lunar surface. This amount of water is equal to about $10^{4}$ times the amount of water we estimate to have been lost per billion years from the lunar surface. Accordingly, it seems reasonable to assert that there should still be detectable amounts of ice in the permanently shaded areas of the moon if the moon has undergone a bulk chemical differentiation as small as about one one-thousandth that of the earth. We may conclude, then, that ice should prove to be a most sensitive mineral indicator of the degree of chemical differentiation of the moon, a far more sensitive guide than the percentage of silica in the surface rocks, for instance.

It should be pointed out that accretions of meteoritic water upon the moon are probably small when compared with the amount of water liberated from the interior of the moon itself. 


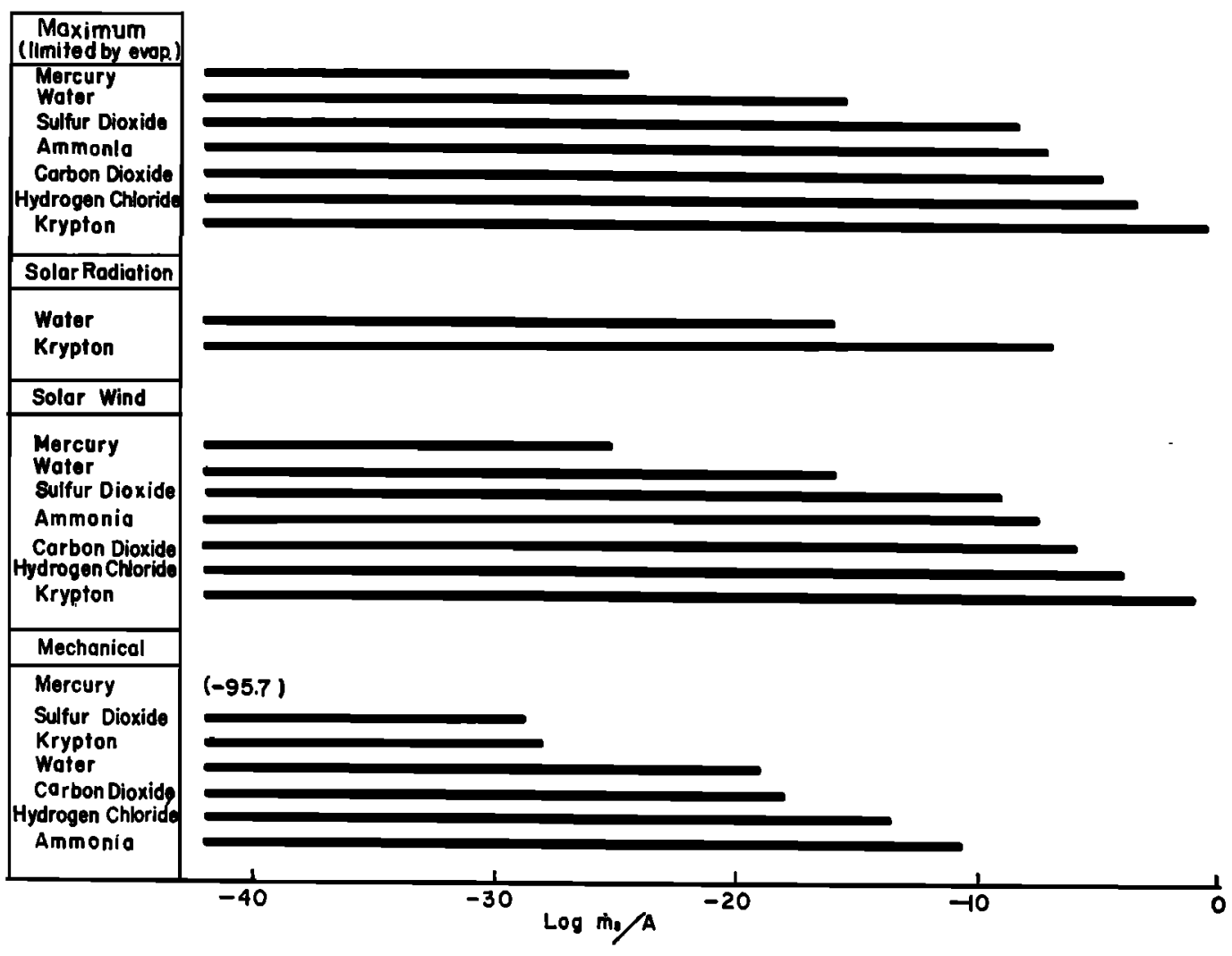

Fig. 4. Mass loss rate in $\mathrm{g} / \mathrm{cm}_{m}{ }^{2} / \mathrm{sec}$ for various escape mechanisms.

The frequency distribution curve for meteoritic masses indicates that at the present rate of influx perhaps 20 grams of meteoritic matter may have been deposited upon every square centimeter of the lunar surface during geologic time [Brown 1960, 1961]. This could have resulted in the liberation of no more than about 0.2 grams of water per square centimeter of the moon, largely from the carbonaceous chondrites.

It should also be pointed out that the flux of 'meteorites' composed primarily of ice of cometary origin is unknown. Such objects might well be fairly abundant, but their very nature would preclude our observing them reliably from the earth's surface.

Finally, it is interesting to compare the required liberation rate of $7 \mathrm{~g} / \mathrm{cm}^{2} /$ billion years computed in the previous section with the thickness of a lunar crust containing 0.03 per cent water which would have to be differentiated to produce that much water. The required thickness is 78 meters, only about one-half of 1 per cent of the average crustal thickness on the earth. 3.6. Validity of migration model. It is now possible to return to the question of the relative distance between cold traps as compared to the average distance covered by a migrating molecule in a single jump. It will be recalled from section 2 that our theory assumes that the beginning and end points of a single jump are uncorrelated-in particular, that a molecule will not find itself in an area devoid of cold traps which is large compared to its average jump length. Actually such a case exists for water molecules that are released in an equatorial area, but we will now show that this departure from our model is not significant. The 'barren' equatorial areas are approximately compensated by the polar areas where the proportion of cold traps is much larger than indicated by our average value of $K$ for the entire moon, and the jump distances are less because of the lower average surface temperature.

The opposite extreme of our uncorrelated 
model would be the case where the jump lengths are so short compared with the separation between cold traps that there is little migration, i.e. that the beginning and end points are highly correlated in latitude. For instance, very few molecules released in the equatorial areas could ever reach the higher latitudes to be trapped. However, molecules released in the polar areas would be confined to these higher latitudes where the larger $K$ would result in a higher proportion being captured. Hence, for the correlated case the loss rate from the cold traps is reduced about 25 per cent (see Table 1). We shall examine the trapping of newly liberated volatiles for the correlated model by considering two cases: uniform liberation over the lunar surface, and the extreme case of liberation from the equatorial areas only.

The uniform liberation results in the loss rate during migration to the cold traps being increased about 50 per cent. The net effect when combined with the lower loss rate from the cold traps is to increase the required liberated flux to balance cold-trap loss by about 10 per cent on the average. Clearly this difference is insignificant in terms of the uncertainties in $K$ and $\alpha$, and the use of the uncorrelated model is sufficiently accurate for our purposes.

The correlated and uncorrelated models do differ significantly, however, in regard to preferential loss of volatiles liberated in the equatorial regions. A rigorous treatment of this problem is involved, but the following analysis" is sufficient to justify the uncorrelated model.

From Table 1 it will be seen that the fraction of permanently shaded area becomes insignificant for latitudes less than about $50^{\circ}$. Accordingly, the worst case for a migrating molecule to reach a cold trap is its release at the equator, $1500 \mathrm{~km}$ from the $50^{\circ}$ latitude zone where the truly uncorrelated model begins to hold. The migration is presumed to be a random walk in arbitrary directions with an average jump distance, for a water molecule, of $280 \mathrm{~km}$ (Table 2). The average component of northerly or southerly migration per jump is then $.707 \bar{D}=200 \mathrm{~km}$. We wish to find the expected number of jumps, $E(N)$, for the molecule to reach either $50^{\circ} \mathrm{N}$ or $50^{\circ} \mathrm{S}$. This condition can be expressed as

$$
\Delta N=\frac{1500}{200}=7.5
$$

where $\Delta N$ is the difference between the number of northerly component and the number of southerly component jumps which comprise $N$. But since the direction of jump is presumed to be random

$$
\Delta N=N^{1 / 2}
$$

and

$$
N=(7.5)^{2}=56
$$

Since on each jump the probability of escape is $\alpha$, the probability of reaching $50^{\circ}$ latitude by random walk without escaping is just

$$
(1-\alpha)^{N}=1-N \alpha
$$

since $N^{2} \alpha^{2}$ is negligible.

From Table 2, $\alpha=4 \times 10^{-3}$

$$
\therefore(1-\alpha)^{N}=0.78
$$

Hence, for the most extreme case, 22 per cent $\dot{m}_{f}$ at the equator would be lost before entering into the uncorrelated zone. The loss decreases for sources located between $0^{\circ}$ and $50^{\circ}$ latitude, and it is clear that the net preferential selection effection is insignificant. Thus, the uncorrelated model is indeed a valid description of migration as well as total trapping of volatiles on the lunar surface.

We have deferred until the present section a discussion of the effect on the mass loss rate of variations in the parameters $K, a$, and $\alpha$. It is clear from equations 8 and 9 that $K$ and $a$ only occur as the product $K a$. If we double the value of $K a$, we increase $\gamma$ from .555 to .714. Thus the total mass loss from the cold traps is increased but the fraction of liberated volatiles lost from the atmosphere is decreased; the net effect is to reduce the required liberated flux to balance the cold trap loss by a factor 2 . If the value of $K a$ is halved, or $\alpha$ is doubled, then $\gamma$ decreases from $\mathbf{. 5 5 5}$ to $\mathbf{. 3 8 5}$, and the net effect is to increase the required liberated flux by a factor 2. Obviously these changes in mass loss are insignificant compared to changes in the evaporation rates with temperature. We have used $120^{\circ} \mathrm{K}$ for the cold trap temperature, with the understanding that it represents a maximum. If a more likely temperature of $100^{\circ} \mathrm{K}$ is used, the mass loss rate of water from the cold traps is reduced by four orders of magnitude, and this loss is insignificant over the lunar lifetime 
(equivalent to a maximum loss from the cold trap surfaces of 2-mm thickness per billion years).

Finally, we can summarize our conclusions from this study by stating that the permanently shaded areas are such an efficient trap that the discovery of no ice in these areas on the moon implies the stringent limitations of an extremely low production of water from the lunar surface during its lifetime.

The atmospheric density that corresponds to the vapor pressure of water at $120^{\circ} \mathrm{K}$ is $3.5 \times 10^{4}$ molecules/ $\mathrm{cm}^{3}$. It is interesting to note at this point that the degree of ionization of water produced by the solar wind is approximately the escape probability $\alpha=4 \times 10^{-3}$, since this represents the fraction of molecules that are ionized and escape; the ion density would be $10^{2} / \mathrm{cm}^{3}$. Clearly this result is compatible with the density of $10^{3}$ electrons/ $\mathrm{cm}^{3}$ observed by Elsmore [1957]. In addition, the logarithmic variation of vapor pressure with inverse temperature implies that the vapor pressure decreases very rapidly for a small temperature decrease. Since $120^{\circ} \mathrm{K}$ is an upper limit, we should actually expect to find even lower densities.

It has been suggested in personal communications to the authors that sputtering [see Wehner, 1955] might be a significant erosional mechanism acting on the ice in the cold traps. Generally the threshold energy for sputtering is at least an order of magnitude greater than the latent heat of vaporization. Recent calculations by Brandt [1961] suggest that Chamberlain's [1961] model of the solar corona with velocities of a few kilometers per second and a density of around 30 electrons $/ \mathrm{cm}^{3}$ at the earth's orbit is in agreement with the deflection of comet tails. The resulting energy flux is many orders of magnitude lower than the radiative heat transfer from the cold trap surfaces and seems to exclude the possibility of sputtering having an appreciable effect on the mass removal rate of ice from the cold traps.

\section{SUMmary}

We have developed a model of the stability of volatiles on the lunar surface. This model is based upon the presumed existence of permanently shaded areas that act as cold traps for volatiles and a rarefied atmosphere that permits the use of simple dynamic trajectories over the lunar surface. Both these assumptions are validated by observations of the lunar surface. The model yields simple expressions for the mass removal rates of volatiles from the cold traps and their migration to the cold traps following release from the lunar crust. In particular:

$$
\dot{m}=(1-\gamma)\left(A K \dot{E}+\dot{m}_{f}\right)
$$

where

$\dot{m}=$ total mass removal rate of a volatile from the moon $(\mathrm{g} / \mathrm{sec})$.

$A=$ surface area of moon $\left(\mathrm{cm}^{2}\right)$.

$K=$ fraction of surface in permanent shadow.

$\dot{E}=$ evaporation rate of volatile at temperature of cold trap (assumed to be $120^{\circ} \mathrm{K}$ in calculation) $\left(\mathrm{g} / \mathrm{cm}^{2} / \mathrm{sec}\right)$.

$\dot{m}_{f}=$ mass influx rate for liberation of new volatile material on lunar surface $(\mathrm{g} / \mathrm{sec})$.

$\gamma=K a(1-\alpha) /[K a(1-\alpha)+\alpha]=$ ratio of trapped fraction to trapped plus escaping fractions.

where

$a=$ the accommodation coefficient of the cold traps.

$\alpha=$ escape probability on a single jump.

The parameters of the model have been examined in order to estimate reasonable and also limiting values for water on the lunar surface. These are:

$$
\begin{aligned}
K= & 5 \times 10^{-3} \text { with a maximum range of } \\
& 1 \times 10^{-3} \text { to } 1 \times 10^{-2} . \\
a & =\text { approximately } 1 . \\
\alpha & =4 \times 10^{-3} \text { for photodissociation of } \\
& \text { water. } \\
\alpha_{\max }= & 1 \text { for maximum loss by evaporation. }
\end{aligned}
$$

The application of the model to common volatiles leads us to the conclusion that only water is relatively stable over periods comparable to the lunar lifetime. We have calculated the total mass loss rate of water from the cold traps to be approximately $4 \mathrm{~g} / \mathrm{cm}^{2} /$ billion years for the reasonable estimate. We also estimated the average liberation rate of water over the lunar surface necessary to condense in the cold traps and balance the loss to be $7 \mathrm{~g} / \mathrm{cm}^{2} /$ billion years. 
Finally we have discussed the validity of our model and the effects of possible variations in the parameters $\alpha, K$, and $a$. The model was found to be quite adequate, since the variation in parameters produced effects which are negligible compared to the change in mass loss rate introduced by reducing the cold trap temperatures from the maximum of $120^{\circ} \mathrm{K}$ to a more reasonable temperature of $100^{\circ} \mathrm{K}$.

These results imply that the lack of ice in the cold traps today would require that the liberation of water from the moon during its lifetime must have been extremely low compared with that on the earth and hence the implication that the chemical differentiation to form a lunar crust has been negligible.

Acknowledgement. This work has been supported in full by the National Aeronautics and Space Administration under Grant NsG 56-60.

\section{REFERENCES}

Allen, C. W., Astrophysical Quantities, Athlone Press, p. 162, 1955.

Barabashov, N. P., A. A. Michailov, V. N. Lipski, editors of Atlas of the Opposite Side of the Moon, Academy of Sciences, USSR, 1961.

Brandt, J. C., On the study of comet tails and models of the interplanetary medium, Astrophys. $J .$, 133, 1091-1092, 1961.

Bridgman, P. W., High pressure effects, in American Institute of Physics Handbook, 1957.

Brown, Harrison, The density and mass distribution of meteoritic bodies in the neighborhood of the earth's orbit, J. Geophys. Research, 65, 1679-1683, 1960.

Brown, Harrison, Addendum: The density and mass distribution of meteoritic bodies in the neighborhood of the earth's orbit, $J$. Geophys. Research, 66, 1316-1317, 1961.

Chamberlain, J. W., Interplanetary gas, 3, A hydrodynamic model of the corona, Astrophys. J., 198, 675-687, 1961.

Dollfus, A., Nouvelle recherche d'une atmosphere au voisinage de la lune, Comptes rendus, Academie des Sciences, 294, 2046-2049, Paris, 1952.

Dollfus, A., Recherche d'une atmosphere autour de la lune, Ann. d'Astrophys., 19, 71, 1956.

Elsmore, B., and G. R. Whitfield, Lunar occultation of a radio star and the derivation of an upper limit for the density of the lunar atmosphere, Nature, 176, 457-458, 1955.

Elsmore, B., Radio observations of the lunar atmosphere, Phil. Mag., 2, 1040-1046, 1957.

Estermann, I., Gases at low densities, in Thermodynamics and Physics of Matter, Vol. 1, High Speed Aerodynamics and Jet Propulsion, edited by
F. D. Rossini, Princeton University Press, pp. 742-744, 1955.

Herring, J. R., and A. L. Licht, Effect of solar wind on the lunar atmosphere, Science, 130, 266, 1959.

International Critical Tables, 9, 201-246, McGrawHill Book Company, New York, 1928.

Jaeger, J.C., The surface temperature of the moon, Australian J. Phy., 6, 10-21, 1953.

Knudsen, M., Die molekulare wärmeleitung der gase und der akkommodationskoeffizient, $A n n$. Physik, 34, 593-656, 1910.

Kuiper, G. P., Planetary atmospheres and their origin, in The Atmosphere of the Earth and Planets, revised edition, edited by G. P. Kuiper, University of Chicago Press, Chicago, p. 367, 1952.

Langmuir, I., The condensation and evaporation of gas molecules, Phys. Rev., 3, 141-147, 1917.

Loeb, L. B., Kinetic Theory of Gases, 1st ed., MeGraw-Hill Book Company, Inc., New York, pp. 240-337, 1927.

Menzel, D. H., Water-cell transmissions and planetary temperatures, Astrophys. $J ., 58,65-74$, 1923.

Öpik, E. J., The density of the lunar atmosphere, Irish Astron. J., $3,137-143,1955$.

Öpik, E. J., and S. F. Singer, Escape of gases from the moon, $J$. Geophys. Research, 65, 3065-3070, 1960.

Pettit, E., Radiation measurements on the eclipsed moon, Astrophys. J., 91, 408-420, 1940.

Pettit, E., and S. B. Nicholson, Lunar radiation and temperatures, Astrophys. J., 71, 102-135, 1930.

Rankama, K., and Th. G. Sahama, Geochemistry, University of Chicago Press, Chicago, 1950.

Sinton, Wm., Observations of solar and lunar radiation at 1.5 millimeters, J. Opt. Soc. Am., 45, 975-979, 1955 .

Spitzer, L., Jr., The terrestrial atmosphere above $300 \mathrm{~km}$, in The Atmosphere of the Earth and Planets, revised edition edited by G. P. Kuiper, University of Chicago Press, Chicago, pp.239-244, 1952.

Tschudin, K., Die Verdampfungsgeschwindigkeit von Eis, Helvetic Physica Acta, 19, 91-102, 1946.

Urey, H. C., The Planets, Their Origin and Development, Yale University Press, New Haven, pp. 17-18, 1952.

Vestine, E. H., Evolution and nature of the lunar atmosphere, Rand Research Memo. RM-2106, 1958.

Watson, K., B. Murray, and Harrison Brown, On the possible presence of ice on the moon, J. Geophys. Research, 66, 1598-1600, 1961.

Wehner, G. K., Sputtering by ion bombardment, in Advances in Electromics and Electron Physics, 7, 239-298, Academic Press, 1955.

Wesselink, A. J., Heat conductivity and the nature of the lunar surface material, Bull Astr. Inst. Netherlands, 10, 351-363, 1948.

(Manuscript received June 9, 1961; revised June 30, 1961.) 